\title{
A probabilistic map of the human ventral sensorimotor cortex using electrical stimulation
}

\author{
Jonathan D. Breshears, MD, ${ }^{1}$ Annette M. Molinaro, $\mathrm{PhD},{ }^{1,2}$ and Edward F. Chang, MD1,3-5 \\ Departments of ${ }^{1}$ Neurological Surgery, ${ }^{2}$ Epidemiology and Biostatistics, and ${ }^{3}$ Physiology, and ${ }^{4}$ Center for Integrative \\ Neuroscience, University of California, San Francisco; and ${ }^{5}$ Center for Neural Engineering and Prostheses, University of \\ California, Berkeley and San Francisco, California
}

\begin{abstract}
OBJECT The human ventral sensorimotor cortex (VSMC) is involved in facial expression, mastication, and swallowing, as well as the dynamic and highly coordinated movements of human speech production. However, vSMC organization remains poorly understood, and previously published population-driven maps of its somatotopy do not accurately reflect the variability across individuals in a quantitative, probabilistic fashion. The goal of this study was to describe the responses to electrical stimulation of the vSMC, generate probabilistic maps of function in the vSMC, and quantify the variability across individuals.
\end{abstract}

METHODS Photographic, video, and stereotactic MRI data of intraoperative electrical stimulation of the vSMC were collected for 33 patients undergoing awake craniotomy. Stimulation sites were converted to a 2D coordinate system based on anatomical landmarks. Motor, sensory, and speech stimulation responses were reviewed and classified. Probabilistic maps of stimulation responses were generated, and spatial variance was quantified.

RESULTS In 33 patients, the authors identified 194 motor, 212 sensory, 61 speech-arrest, and 27 mixed responses. Responses were complex, stereotyped, and mostly nonphysiological movements, involving hand, orofacial, and laryngeal musculature. Within individuals, the presence of oral movement representations varied; however, the dorsal-ventral order was always preserved. The most robust motor responses were jaw (probability 0.85), tongue (0.64), lips (0.58), and throat (0.52). Vocalizations were seen in 6 patients (0.18), more dorsally near lip and dorsal throat areas. Sensory responses were spatially dispersed; however, patients' subjective reports were highly precise in localization within the mouth. The most robust responses included tongue (0.82) and lips (0.42). The probability of speech arrest was 0.85 , highest $15-20 \mathrm{~mm}$ anterior to the central sulcus and just dorsal to the sylvian fissure, in the anterior precentral gyrus or pars opercularis.

CONCLUSIONS The authors report probabilistic maps of function in the human vSMC based on intraoperative cortical electrical stimulation. These results define the expected range of mapping outcomes in the vSMC of a single individual and shed light on the functional organization of the vSMC supporting speech motor control and nonspeech functions.

http://thejns.org/doi/abs/10.3171/2014.11.JNS14889

KEY WORDS sensorimotor cortex; corticobulbar; brain mapping; probabilistic maps; diagnostic and operative techniques

$\mathrm{O}$ VER 70 years ago, Penfield (1937) published his seminal work on the somatotopy of the human peri-rolandic cortex, fortifying the notion of the classic sensorimotor homunculus. Surprisingly, few additional investigations with direct electrocortical stimulation (ECS) have been undertaken to study the organization of the ventral half of the sensorimotor cortex (VSMC) in humans, where speech articulators and nearby language centers make this area unique. ${ }^{1,4}$ Neuroanatomically, the $\mathrm{vSMC}$ is distinct from the dorsal sensorimotor cortex (dSMC). Its neurons project via the corticobulbar pathway to synapse in the brainstem motor nuclei, providing bilateral innervation to the muscles of the upper face, jaw, oropharynx, and vocal tract through cranial nerves (CNs)

ABBREVIATIONS AIC = Akaike information criterion; $A P=$ anterior-posterior; $C N=$ cranial nerve; $C S=$ central sulcus; $d S M C=$ dorsal $S M C ; D V=$ dorsal-ventral; $E C o G$ = electrocorticography; ECS = electrocortical stimulation; fMRI = functional MRI; MNI = Montreal Neurological Institute; $S F=$ sylvian fissure; $S M C=$ sensorimotor cortex; $\mathrm{VSMC}=$ ventral SMC.

SUBMITTED April 23, 2014. ACCEPTED November 7, 2014.

INCLUDE WHEN CITING Published online May 15, 2015; DOI: 10.3171/2014.11.JNS14889.

DISCLOSURE Dr. Chang was funded by US National Institutes of Health grants R01-DC012379, R00-NS065120, and DP2-OD00862 and the Ester A. and Joseph Klingenstein Foundation. The authors report no conflict of interest concerning the materials or methods used in this study or the findings specified in this paper. 
V, VII, IX, X, and XI. In humans, there is an additional ipsilateral pathway from the cortical laryngeal representation through the nucleus ambiguus and CN IX, X, and $\mathrm{XI}$ to subserve phonation and the laryngeal musculature that is not present in nonhuman primates..$^{10-12}$ The lower facial muscles innervated by CN VII and the muscles of the tongue innervated by CN XII also receive only unilateral innervation from the contralateral vSMC. The human vSMC and corticobulbar tract appear uniquely evolved for both the basic functions of facial expression, mastication, and swallowing, as well as the dynamic and highly coordinated movements of human speech production. ${ }^{10}$ Thus, an understanding of vSMC organization and variability among individuals is especially salient for the study of speech production.

Before Penfield's work, initial inferences about the existence and organization of a motor area were made by Hughlings Jackson based on behavioral observations of seizure propagation in epilepsy patients. ${ }^{6}$ Hitzig and Ferrier were the first to confirm these inferences in animals using galvanic current. ${ }^{5,7}$ Foerster gave the first detailed description of motor organization in humans-later popularized by Penfield. ${ }^{6,17}$ In this popular conception of vSMC organization, there is a discretely ordered progression of representations for the lips, jaw, tongue, and pharynx/ larynx, respectively, along the dorsal-to-ventral orientation of the central sulcus. More contemporary electrical stimulation $^{8,9,15,23}$ and functional MRI (fMRI) ${ }^{14,21}$ studies in humans and monkeys have demonstrated that Penfield's original somatotopy holds true in general; however, ECS in individual patients rarely fully recapitulates what was described in those early published maps.

The expected results of stimulation of the vSMC in an individual patient have never been described in a quantitative, probabilistic fashion that accurately reflects the variability across individuals. Focusing on the vSMC of the dominant hemisphere, we sought to answer several important questions. What behavioral responses can be evoked by stimulation, and what is their frequency of observation and spatial variance in the population? In a single individual, what is the probability of observing a given motor or sensory response, and what cortical location is most likely to render that response when stimulated?

\section{Methods \\ Subjects}

This study was approved by the Committee on Human Research of the University of California, San Francisco. Inclusion criteria included awake craniotomy with motor and speech mapping of the dominant hemisphere for resection of a lesion (tumor, arteriovenous malformation, or epileptic foci). Exclusion criteria included gross cortical dysplasia, significant mass effect/cortical distortion from the underlying lesion, and insufficient extent or density of mapping (less than 4 positive stimulation sites).

\section{Intraoperative Mapping}

After exposure of the peri-rolandic cortex and emergence from intravenous sedation, intravenous fentanyl was titrated for optimal balance of pain control with pa- tient arousal during the mapping procedure. The vSMC was completely and densely mapped (by E.F.C.) using an Ojemann stimulator (current range: 1-3.5 mA, pulse frequency $60 \mathrm{~Hz}$, pulse width $1 \mathrm{msec}$, stimulus duration: 500-1500 msec, stimulator electrode spacing $5 \mathrm{~mm}$ ). To minimize the occurrence of false-negative mappings, the stimulation current was incrementally increased until a motor response was obtained in each patient at the beginning of the mapping procedure. Each response to stimulation was tested for consistency/repeatability by stimulating each cortical site 3 times nonsequentially. Sites were considered positive if the same response was elicited on all 3 trials. Responses were considered valid only in the absence of afterdischarges or seizure activity on electrocorticography (ECoG), which was monitored and reported in real time by an epileptologist. During portions of the mapping, patients were asked to protrude their tongues during stimulation to assess for tongue movements. During testing for speech arrest, patients were asked to count to 30 , recite the days of the week, repeat multisyllabic words, and name pictures. In 29 of 33 cases, synchronized high-definition video (and microphone audio) recordings of the exposed brain and the patient's face were taken for the duration of the mapping procedure. Photographs were taken orthogonally to the exposed cortical surface after the completion of the mapping procedure to document the stimulation sites. Finally, in 20 cases, the stimulation sites were co-registered with the patient's MRI using a Brainlab stereotactic navigation system.

\section{Measurement of Stimulation Sites}

All stimulation sites were measured in a common 2D cortical surface coordinate system (Fig. 1). First, the central sulcus (CS) and sylvian fissure (SF) were traced on the photographs, using the MR-based cortical surface reconstructions to help identify these landmarks. Second, the CS was approximated with a straight line extending from the SF to the most dorsal aspect of the craniotomy exposure. For each stimulation site, 2 measurements were made. An anterior-posterior (AP) measurement was made from the stimulation site to the CS. This AP measurement was orthogonal to the linear approximation of the CS. Second, a dorsal-ventral (DV) measurement was made along the linear approximation of the CS from the level of the stimulation site to the SF. AP measurements anterior to the CS and DV measurement above the SF were defined as positive. This method allowed stimulation sites across all patients to be combined into a common 2D reference space with reproducibility while minimizing distortion due to variability in cortical anatomy among individuals.

\section{Classification of Motor and Sensory Responses}

Intraoperative video was reviewed, and patient motor and sensory responses were described. When video was not available (4 patients), intraoperative transcripts made at the time of mapping were used. This led to the identification of 8 groups of motor responses (eye, arm, hand, lips/mouth, jaw, tongue, throat, and vocalization). Within groups, multiple stereotyped responses were often seen across patients. Therefore, all motor responses to stimula- 

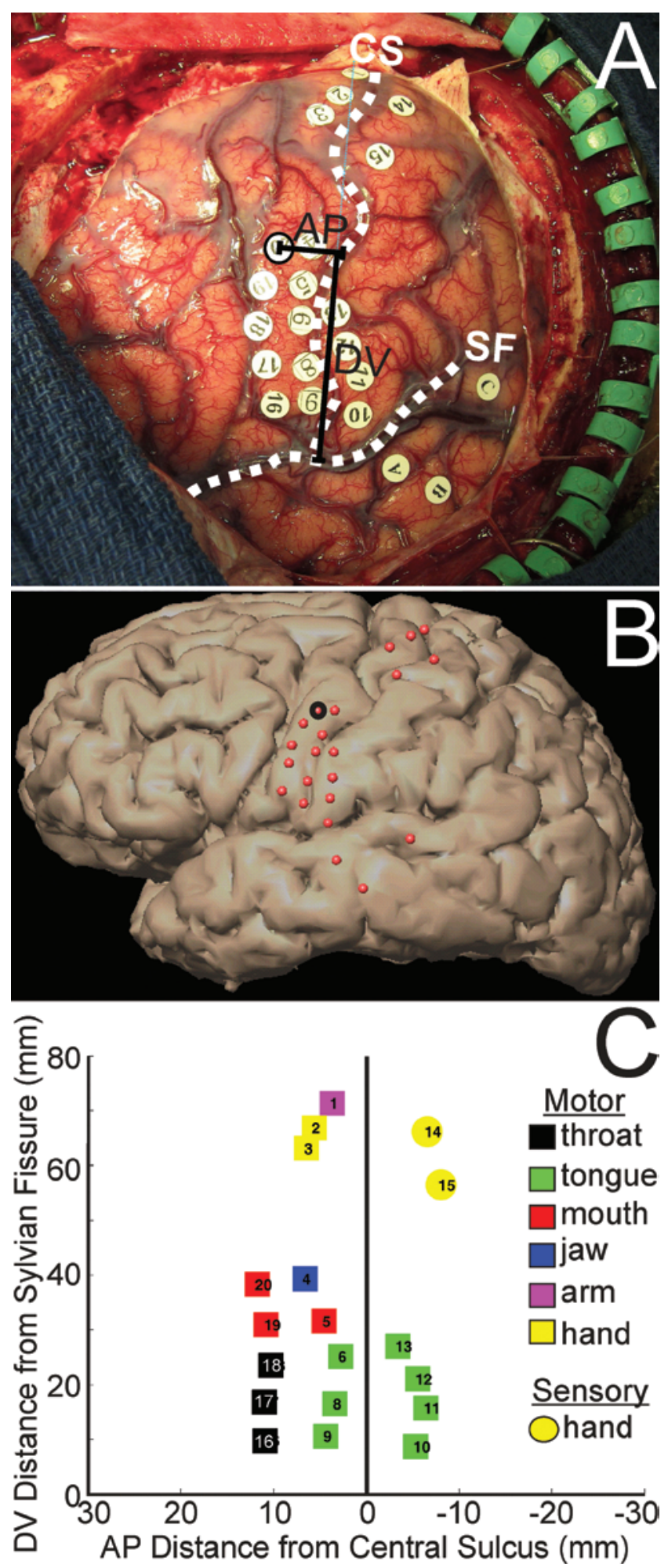

FIG. 1. Measurement of stimulation sites. The intraoperative photograph, which was obtained after the completion of cortical mapping with electrical stimulation, shows examples of anterior-posterior (AP) and dorsal-ventral (DV) measurements for Site 20 (A). The central sulcus (CS) and sylvian fissure (SF) are identified with the assistance of stereotactic neuronavigation (B) and traced on the photograph (white dashed lines). All stimulation response sites for the individual are plotted in the 2D AP, DV coordinate system (C). Negative values are posterior to the CS. Figure is available in color online only. tion were classified according to these observations. Due to the variable dorsal extent of the craniotomies, there were insufficient eye (4 sites) and arm (5 sites) responses, and these 2 groups were not included in further analysis. Patients' sensory responses (patient descriptions of their sensory perceptions) were similarly classified according to the 7 categories of sensory responses that were reported by patients.

\section{Probabilistic Maps of Function}

For each response category (hand, lips, jaw, etc.), a 2D spatial histogram spanning $60 \mathrm{~mm} \mathrm{AP}$, centered on the $\mathrm{CS}$, and extending $80 \mathrm{~mm}$ dorsal to the SF was created with a bin size of $5 \mathrm{~mm}^{2}$. Responses in each bin were weighted such that a single patient's total contribution to a histogram for a particular response category equaled 0 (no response) or 1 (response). This was accomplished by dividing by the number of responses observed from that particular patient. For example, if a patient had tongue movements elicited from 2 cortical sites, then each site received a weight of 0.5 . These weighted responses in each bin were then summed across all patients and divided by the total number of patients stimulated in the cortical area covered by that bin. Because dense mapping was performed to the full spatial extent of the craniotomy in each case, this denominator was determined by the dorsal extent of each patient's craniotomy, which was dictated by clinical indications (see Table 1). This gave the probability of observing a given response type at a particular cortical location in an individual patient, as shown in the following equation:

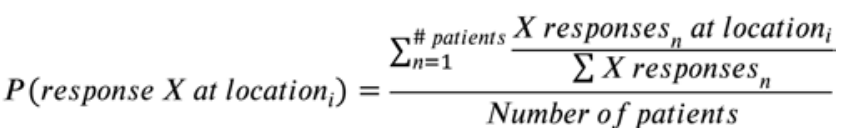

The overall probability of observing that response at any cortical location in an individual was thus equal to the integral over the entire probability map.

This probabilistic histogram was then smoothed with linear interpolation and remapped back onto a standard Montreal Neurological Institute (MNI) template brain for visualization purposes. These maps were built and evaluated by iteratively resampling the stimulation sites 100 times, adjusting for repeated measures by selecting only one site from each patient to create a "training data set." From each training set, a corresponding probability map was created. The remaining unselected stimulation sites were then used as a "test set." The mean Euclidian distance between each observation in the test set and the location with maximum probability in the training map was calculated for each iteration. Finally, all training maps were combined to calculate a mean probability map, the standard deviation of the map, as well as the average Euclidian error against the test sets as a measure of spatial variability.

\section{Determination of DV Order}

To determine the DV ordering of motor and sensory responses, the distributions of DV locations for each re- 
TABLE 1. Demographic and clinical characteristics of 33 patients undergoing awake craniotomy with intraoperative mapping

\begin{tabular}{|c|c|c|c|c|}
\hline Case No. & Sex & Age (yrs) & Diagnosis & Dorsal Extent of Craniotomy $(\mathrm{mm})$ \\
\hline 1 & M & 37 & Anaplastic oligodendroglioma, WHO Grade III & 79.5 \\
\hline 2 & $\mathrm{~F}$ & 23 & Oligoastrocytoma, WHO Grade II & 70.9 \\
\hline 3 & M & 23 & MTS & 38.8 \\
\hline 4 & $\mathrm{~F}$ & 47 & Left hippocampal GBM & 38.9 \\
\hline 5 & M & 42 & Ganglioglioma & 54.1 \\
\hline 6 & M & 65 & LGG & 72.2 \\
\hline 7 & $\mathrm{~F}$ & 30 & Anaplastic astrocytoma & 33.5 \\
\hline 8 & $\mathrm{~F}$ & 20 & Epilepsy & 43.6 \\
\hline 9 & M & 43 & MTS & 52.6 \\
\hline 10 & M & 54 & Cavernous malformation & 51.8 \\
\hline 11 & M & 37 & Left temporal epilepsy & 41.2 \\
\hline 12 & $\mathrm{~F}$ & 19 & Anaplastic astrocytoma & 37.1 \\
\hline 13 & M & 36 & Oligoastrocytoma & 33.1 \\
\hline 14 & $\mathrm{~F}$ & 69 & GBM & 78.1 \\
\hline 15 & $\mathrm{M}$ & 27 & Left frontal insular glioma & 72.6 \\
\hline 16 & $\mathrm{~F}$ & 52 & Cavernous malformation & 50 \\
\hline 17 & $\mathrm{~F}$ & 65 & Cavernous malformation & 46.5 \\
\hline 18 & M & 64 & Anaplastic oligodendroglioma & 58 \\
\hline 19 & M & 38 & Ganglioglioma & 40.1 \\
\hline 20 & $\mathrm{~F}$ & 23 & Ganglioglioma & 36.4 \\
\hline 21 & M & 25 & Cavernous malformation & 30.2 \\
\hline 22 & $\mathrm{M}$ & 50 & Oligodendroglioma & 49.2 \\
\hline 23 & $\mathrm{~F}$ & 39 & MTS & 53.2 \\
\hline 24 & $\mathrm{~F}$ & 29 & Left ATL epilepsy & 42 \\
\hline 25 & M & 71 & GBM & 38.1 \\
\hline 26 & $\mathrm{M}$ & 64 & GBM & 46 \\
\hline 27 & $\mathrm{~F}$ & 20 & MTS & 51.1 \\
\hline 28 & $\mathrm{~F}$ & 49 & Oligodendroglioma & 40.2 \\
\hline 29 & $\mathrm{M}$ & 51 & Left temporal GBM & 32.4 \\
\hline 30 & M & 61 & GBM & 52.1 \\
\hline 31 & $\mathrm{~F}$ & 49 & Gliosarcoma, WHO Grade IV & 43.9 \\
\hline 32 & $\mathrm{~F}$ & 49 & Anaplastic astrocytoma, WHO Grade III & 29.1 \\
\hline 33 & $\mathrm{~F}$ & 58 & Glioblastoma, WHO Grade IV & 28.5 \\
\hline
\end{tabular}

$\mathrm{ATL}=$ anterior temporal lobe; $\mathrm{GBM}=$ glioblastoma; $\mathrm{LGG}=$ low-grade glioma; $\mathrm{MTS}$ = mesial temporal sclerosis.

sponse category were compared with an analysis of variance. This was followed by paired comparisons of each distribution with Bonferroni correction. One distribution qualitatively appeared to be bimodal, therefore the distribution was iteratively fit with 1D Gaussian mixture models of varying component number (1-4) to identify the number of components which minimized the Akaike information criterion (AIC). All data analysis was performed in Matlab.

\section{Results}

\section{Patients}

Of 38 patients meeting the inclusion criteria, 5 were excluded for deafness (1), dysplasia (1), mass effect (1), and insufficient extent of mapping (2). Of the remaining 33 pa- tients, 13 were female. The patients' mean age was 38.5 years. (See Table 1 for a summary of demographic and clinical characteristics.) There were 494 positive stimulations sites, including 194 pure motor, 212 pure sensory, 61 speech arrest, and 27 mixed responses (Fig. 2).

\section{Motor Responses}

Motor responses were complex, involving more than 1 muscle group. Movements also appeared unnatural and nonbehavioral. They were observed in all patients for an overall probability of 1 and a maximum probability 0.07 within $5 \mathrm{~mm}$ of the CS and 25-30 $\mathrm{mm}$ dorsal to the SF (Fig. 3A). Excluding those corresponding to eye and arm movements, the remaining motor responses fell into 6 groups: hand (24), lips (61), jaw (59), tongue (58), throat (37), and vocalization (8). 

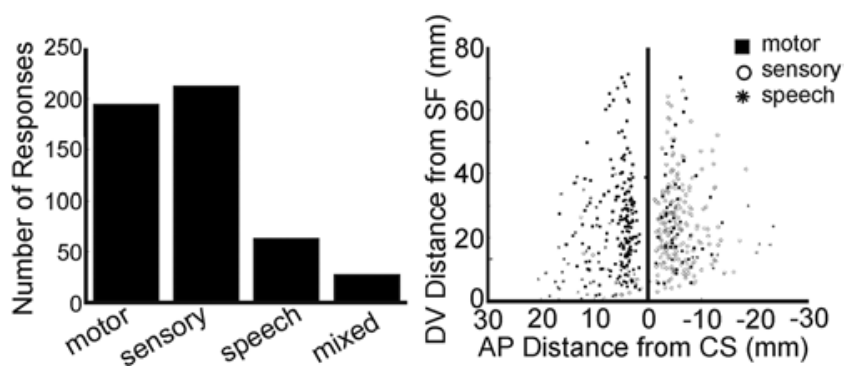

FIG. 2. Left: Bar graph showing response types from all 33 patients.

Right: Graph showing all motor, sensory, and speech sites plotted in the common 2D AP, DV coordinate system.
Hand movements were stereotyped and most often involved flexion of the fingers and wrist. Hand responses were observed in 6 of the 33 patients, for an overall probability of 0.18 ; however, they were seen in 5 of 13 patients with an extent of craniotomy of at least $50 \mathrm{~mm}$ dorsal to the SF (probability 0.38). The maximum probability of observing a hand response was 0.03 within $5 \mathrm{~mm}$ anterior to the CS, between 35 and $40 \mathrm{~mm}$ dorsal to the SF (error 19 $\mathrm{mm}$ ) (Fig. 3A). Because hand is the most dorsal functional group included in this analysis, these results are likely to be heavily affected by the limited dorsal extent of the craniotomy in some cases. Nonetheless, the sites that we were able to record from serve as good reference points for the boundary between the corticospinal and corticobulbar representations (dSMC vs vSMC).

Lip and/or mouth movements were observed in 19 of

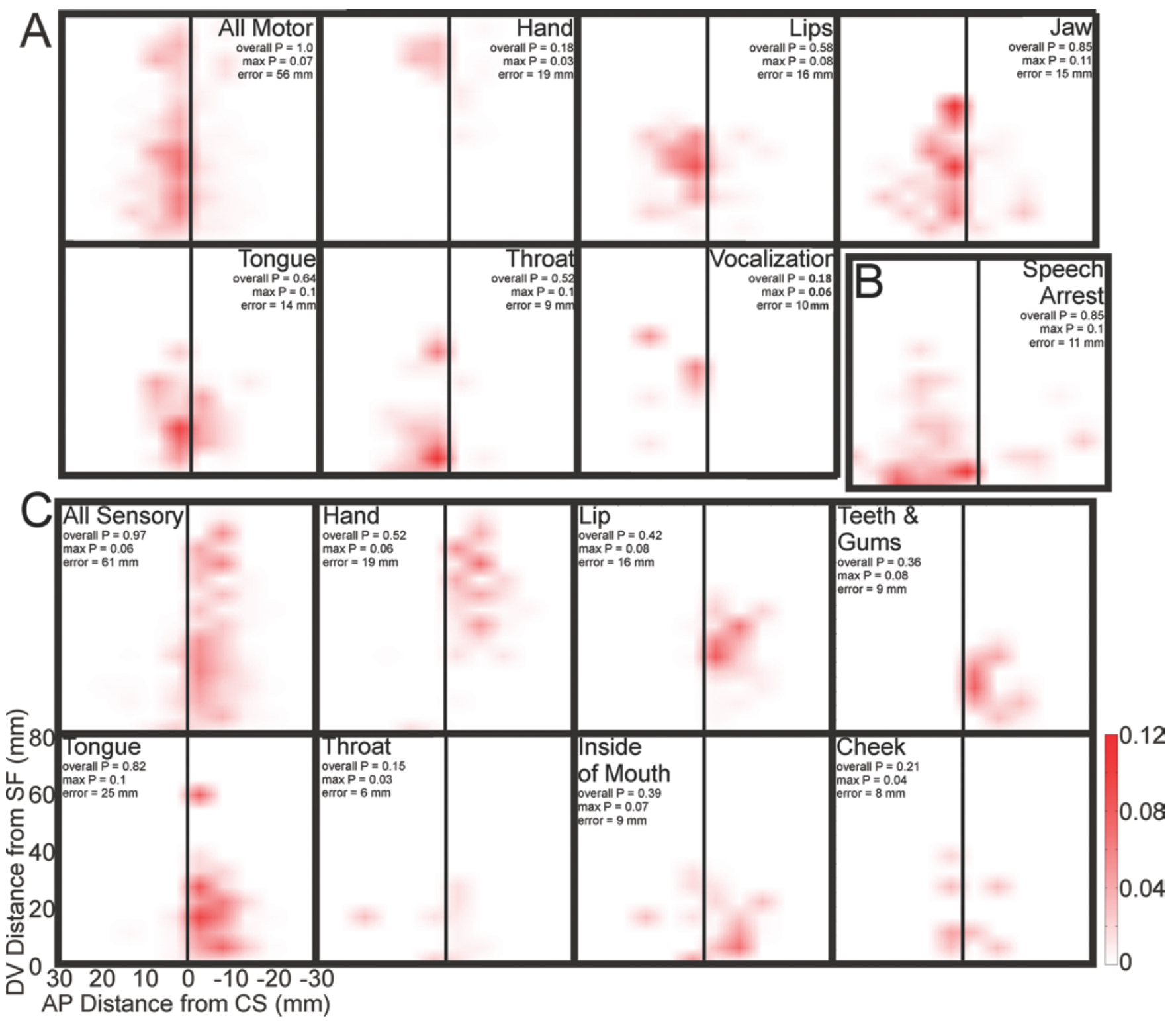

FIG. 3. Maps of the vSMC demonstrating the probability of observing a particular motor (A), speech arrest (B), or sensory (C) response to electrical stimulation at a particular cortical site in an individual patient. $\mathrm{P}=$ probability. Figure is available in color online only. 
the 33 patients (overall probability 0.58 ), with a maximum probability of 0.08 for sites located $0-5 \mathrm{~mm}$ anterior to the $\mathrm{CS}$ and 25-30 $\mathrm{mm}$ dorsal to the SF (error $16 \mathrm{~mm}$ ) (Fig. $3 \mathrm{~A})$. The most frequent response was a stereotyped inferolateral pulling of the contralateral corner of the mouth (muscles: depressor anguli orsi, platysma), which ranged in degree from subtle movement of the corner of the mouth to severe facial distortion and head tilt, occasionally appearing to involve the sternocleidomastoid muscle. The strength of this response tapered off as stimulation moved away from a single "hot spot." A less common recurring response involved the lips coming together or pursing (orbicularis oris). Response sites were contiguous in 13 patients and frequently involved mixed articulators, including the jaw, tongue, or throat.

Jaw movements were observed in 28 of the 33 patients (overall probability 0.85 ), making it the most robust response overall. The maximum probability of observing a response at a single site was 0.11 for sites located $0-5 \mathrm{~mm}$ anterior to the CS and 25-30 mm dorsal to the SF (error $15 \mathrm{~mm}$ ) (Fig. 3A). Typical jaw movements included mandibular depression, elevation (masseter, temporalis, medial pterygoid), contralateral excursion (medial and lateral pterygoids), protrusion (masseter, lateral pterygoid), and retrusion (temporalis, lateral pterygoid). Movements were observed in conjunction with the mouth in 9 patients, the throat in 2 patients, and the tongue in 1 patient.

Tongue movements were observed in 21 of the 33 patients (overall probability 0.64), with a maximum probability of 0.1 for sites located $0-5 \mathrm{~mm}$ anterior to the CS and 15-20 $\mathrm{mm}$ dorsal to the SF (error $14 \mathrm{~mm}$ ) (Fig. 3A). Tongue was the second most robust motor response observed. Responses were observed by having the patient protrude their tongue prior to stimulation. The most common movement was retraction (styloglossus), followed by contralateral deviation (hyoglossus). A frequent observation by patients was a sensation of "freezing" in their mouth or inability to speak.

Throat movement responses were observed in 18 patients (overall probability 0.52 ), with a maximum probability of 0.11 for sites located $0-5 \mathrm{~mm}$ anterior to the CS and 5-10 $\mathrm{mm}$ dorsal to the SF (error $9 \mathrm{~mm}$ ) (Fig. 3A). Responses were described as an inability to breathe, tightening of the throat (laryngeal adductors), or inability to swallow (pharyngeal constrictors). These responses appeared to be bimodal in their DV location, with a ventral throat area just above the SF and a second throat area dorsal to the mouth, jaw, and tongue areas and below the hand area, between 40 and $45 \mathrm{~mm}$ dorsal to the SF. Testing for multimodality by iterative fitting with $1 \mathrm{D}$ Gaussian mixture models of varying component number found that a combination of 2 Gaussians minimized the AIC (AIC 282.5, relative likelihood 0.002). Clear dorsal throat responses were obtained in 8 patients and ventral throat responses in 16, with 6 of the patients having responses in both areas separated by areas of mouth, jaw, or tongue responses.

Vocalization occurred in 6 patients (overall probability 0.18 , maximum probability 0.06) (Fig. 3A). This was characterized by an involuntary forced exhalation (diaphragm), collapsing of the chest, partial tensing of the lips and mouth, and continuous phonation of a vowel-like sound, /aaa.../ (lateral cricoarytenoids, oblique and transverse arytenoids). This response was predominantly evoked in an area superior to lips and always on the anterior aspect of the precentral sulcus (error $10 \mathrm{~mm}$ ).

\section{Speech Arrest}

Disruption of speech with stimulation was observed in 27 patients. The most likely site for speech arrest was seen in the posteriormost Broca's area, including the pars opercularis and part of the precentral gyrus, 15-20 mm anterior to the CS, just above the $\mathrm{SF}$ (probability 0.09 , error 10 $\mathrm{mm})$. Speech arrest sites were also seen more dorsally. The possibility of a motor component to speech arrest in these dorsal areas cannot be ruled out.

\section{Sensory Responses}

Sensory responses were obtained in 32 of 33 patients (overall probability 0.97 ), with a maximum probability of 0.06 for sites located $0-5 \mathrm{~mm}$ posterior to the CS and 20 $25 \mathrm{~mm}$ dorsal to the SF. Sensory responses were almost uniformly described by patients as "tingling" sensations of the hand (44), lips (44), teeth/gums (19), tongue (102), inside of the mouth (27), cheek (9), or throat (10). Patients were frequently able to localize these sensations with high precision (e.g., "tingling in my right upper lip"). There were no reports of a sensation of movement in the absence of actual movement (e.g., stimulation-evoked proprioception). The largest and most frequently identified sensory area was tongue, which was observed in 26 patients (overall probability 0.82 ) with a maximum probability of 0.1 for sites located 0-5 $\mathrm{mm}$ posterior to the CS and 15-20 $\mathrm{mm}$ dorsal to the SF (error $25 \mathrm{~mm}$ ) (Fig. 3C). This was followed by hand in 17 patients (overall probability 0.52 ), with a maximum probability of 0.6 for sites located 5-10 $\mathrm{mm}$ posterior to the $\mathrm{CS}$ and $35-40 \mathrm{~mm}$ dorsal to the SF. A lip area was present in 14 patients (overall probability 0.42 ), with a maximum probability of 0.08 for sites located 0-5 $\mathrm{mm}$ posterior to the CS and $25-30 \mathrm{~mm}$ dorsal to the SF. Teeth/gums, inside of mouth, cheek, and throat had less pronounced responses to cortical stimulation, with overall and maximum probabilities of 0.36 and $0.08,0.39$ and $0.07,0.21$ and 0.04 , and 0.15 and 0.03 , respectively (Fig. 3C).

\section{Dorsal-Ventral Organization}

The order of motor areas, from dorsal to ventral, was hand (median distance from SF 59 mm, 95\% CI 52-63 $\mathrm{mm}$ ), vocalization (33 $\mathrm{mm}, 95 \%$ CI 28-39 mm), mouth (28 mm, 95\% CI 26-31 mm), jaw (22 mm, 95\% CI 19$25)$, tongue (19 mm, 95\% CI 17-22 mm), and throat (12 $\mathrm{mm}, 95 \%$ CI 8-17 mm). As shown in Fig. 4, not all motor groups were observed in every individual. Additionally, the absolute position is variable between subjects. However, no violations of the sequence of somatotopic organization were observed.

There was more overlap of DV location distributions for sensory areas (Fig. 4). The ordering from dorsal to ventral was hand (median distance from SF $44 \mathrm{~mm}, 95 \% \mathrm{CI}$ 39-48 mm), then lip (29 mm, 28-33 mm), followed, in no particular order, by tongue (17 $\mathrm{mm}, 95 \%$ CI $15-19 \mathrm{~mm})$, 

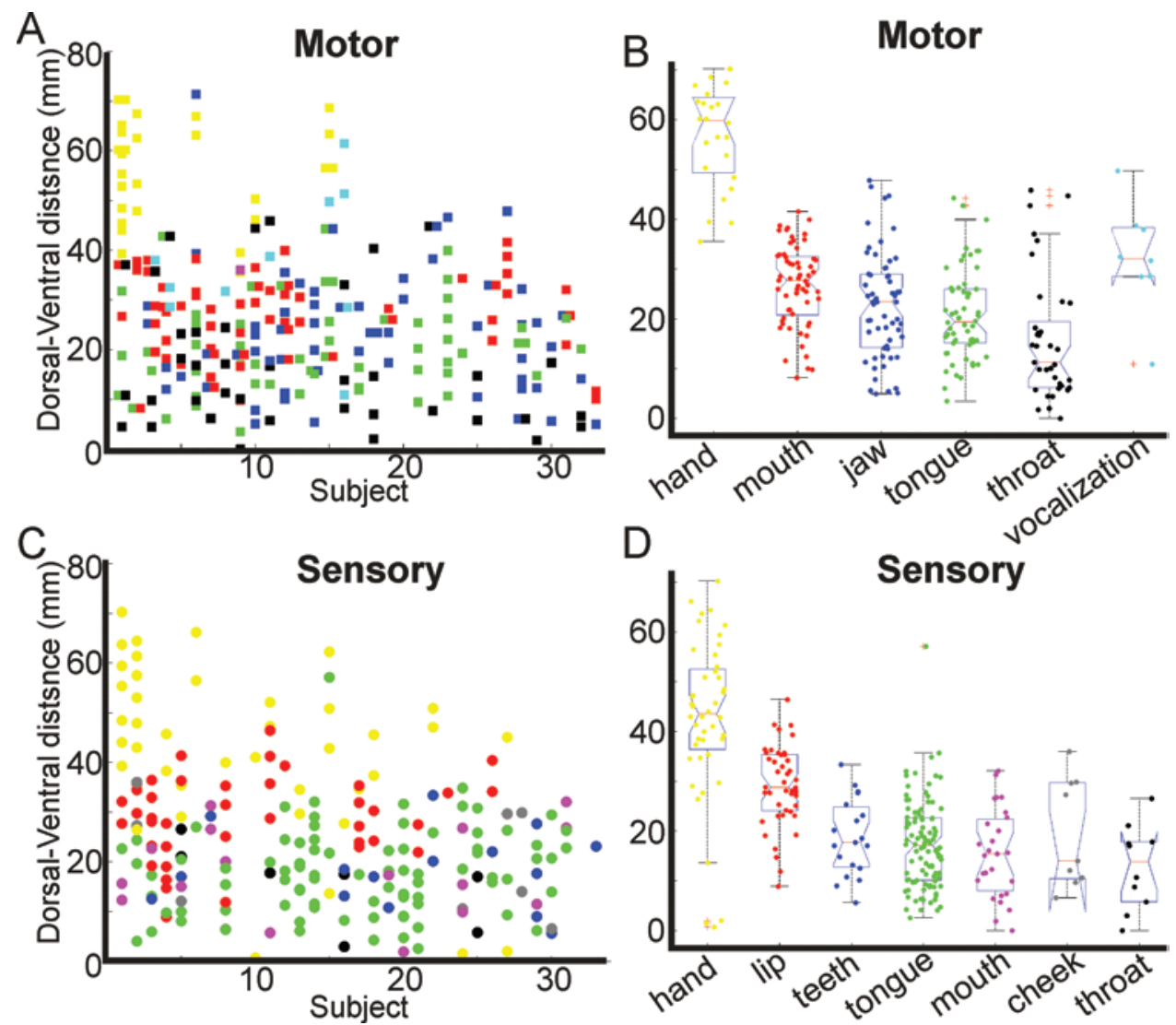

FIG. 4. Motor (A) and sensory (C) responses to stimulation are shown in DV order for 33 individual patients. Not all response types are present in each individual, but the DV order is preserved. The population distributions of DV locations are shown for motor (B) and sensory responses (D). The central mark is the median, the edges of the box are the 25th and 75th percentiles, the whiskers extend to the most extreme data points not considered outliers, and the notches indicate the $95 \%$ confidence interval of the median. Figure is available in color online only.

teeth/gums (18 mm, 95\% CI 13-23 mm), inside of mouth (15 mm, 95\% CI 10-20 mm), cheek (18 mm, 95\% CI 11$30 \mathrm{~mm}$ ), and throat (13 $\mathrm{mm}, 95 \% \mathrm{CI} 9-19 \mathrm{~mm})$.

\section{Discussion}

Stimulation mapping in a single individual rarely recapitulates Penfield's homunculus in its entirety. Rather, motor and sensory responses observed in one individual may be completely absent in another. In his series of 126 patients, Penfield reported only 16 tongue motor sites, 21 lip motor sites, and 41 jaw movement sites ${ }^{17}$ Further, in a single individual, the response to stimulation is not stable; rather, prior stimulations can lead to facilitation or extinction of a response. ${ }^{19}$ In this study, we characterized individual variability across a cohort of patients by providing a granular probabilistic description of evoked behavioral responses from stimulation in the vSMC.

Our results show strong concordance with what is known about the organization of the vSMC (Fig. 5). The responses we observed were not those of voluntary natural movements or sensations and were more similar to those observed during focal seizures (despite the absence of afterdischarges or seizures on ECoG). One possible explanation is that these responses to supraphysiological currents reveal intrinsic "synergies" in muscle coordination. Graziano et al. endorsed this hypothesis after microstimulation experiments in monkey SMC resulted in complex, behaviorally relevant movements. ${ }^{8}$ Further support comes from work by Overduin et al., demonstrating that microstimulation-evoked electromyographic patterns in macaques can be decomposed into smaller sets of muscle synergies that closely mirror those generated by natural hand movements. ${ }^{16}$ Whether our observed movements are behaviorally relevant is unclear. It seems likely that the spatial scale of clinical stimulation $(5 \mathrm{~mm})$ might result in movements closer to the tonic/seizure end of the spectrum, whereas microstimulation $(\sim 1 \mathrm{~mm})$ may produce finer movements closer to the behavioral end of the spectrum. ${ }^{8,9,16}$ Just as in previous human and animal stimulation studies, the observed responses were stereotyped across subjects, allowing for easy classification in most cases.

Throat motor responses are difficult to observe and thus challenging to classify. Despite having to rely on patient reports, our data revealed 2 throat areas that correspond with prior stimulation and fMRI studies. ${ }^{3,20}$ In addition to the ventral "swallow" area originally described by Foerster and Penfield, ${ }^{6}$ we also observe a dorsal throat area, stimulation of which resulted in patients feeling unable to speak or breathe. By having patients perform both vocal 

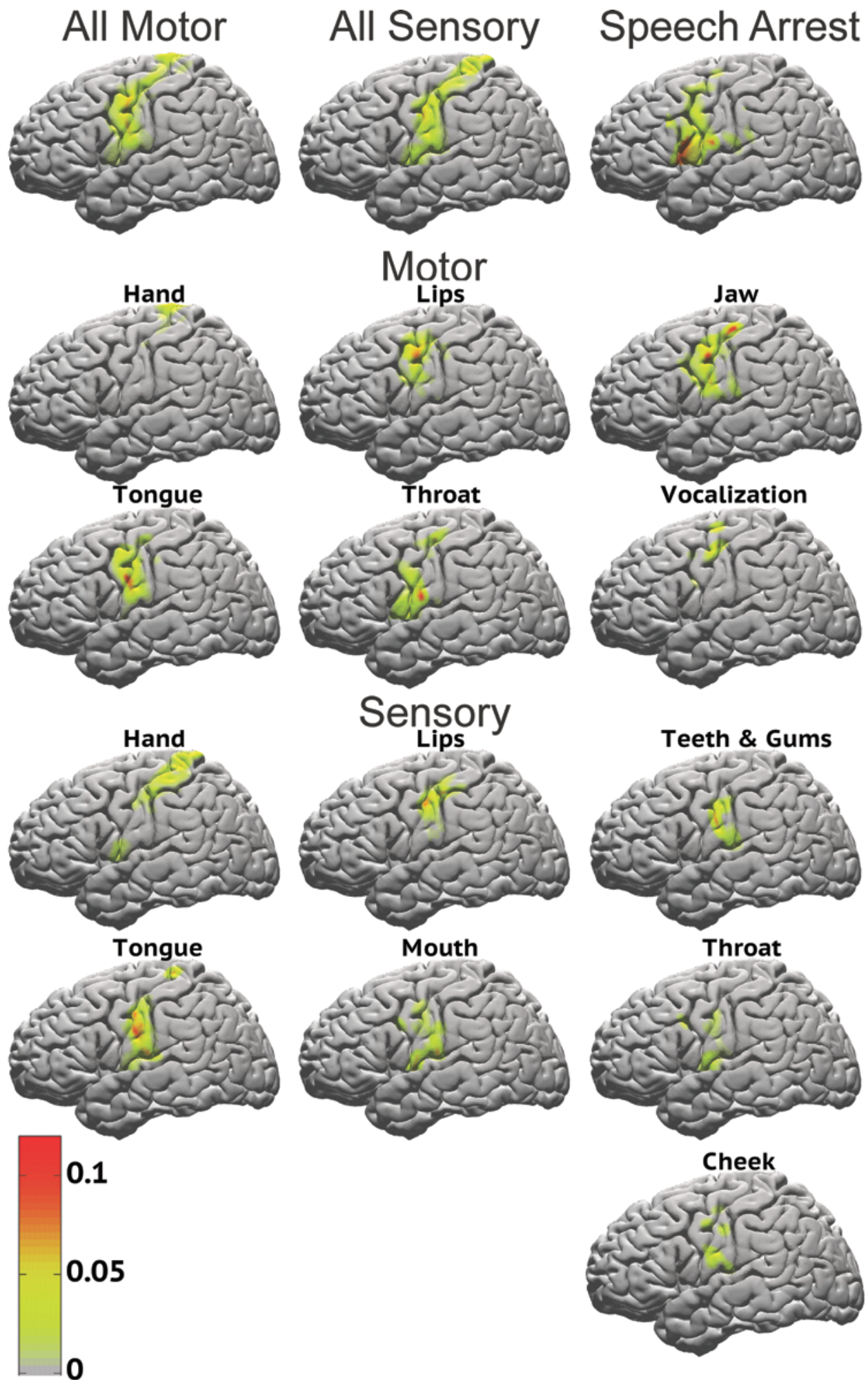

FIG. 5. Probabilistic maps of function as in Fig. 3, transferred onto standard MNI brain surface. Infrasylvian sensory areas are artifacts of the transformation of 2D probability maps in Fig. 3 (which do not include an infrasylvian area along the y-axis) onto the 3D MNI template brain. The maximum and total probability values for all motor, all sensory, and speech arrest areas are, respectively, 0.07 and $1,0.06$ and 0.97 , and 0.09 and 0.82 . The corresponding values for the individual motor areas are 0.023 and 0.18 for hand, 0.08 and 0.58 for lips, 0.11 and 0.85 for jaw, 0.1 and 0.64 for tongue, 0.1 and 0.52 for throat, and 0.06 and 0.18 for vocalization. The corresponding values for the individual sensory areas are 0.06 and 0.52 for hand, 0.8 and 0.42 for lips, 0.8 and 0.36 for teeth and gums, 0.1 and 0.82 for tongue, 0.07 and 0.39 for mouth, 0.03 and 0.15 for throat, and 0.04 and 0.21 for cheek. The color scale shows probability values ranging from 0 to 0.1 . Figure is available in color online only. 
and nonvocal laryngeal tasks, Liotti et al. demonstrated activations in this same area, which they called the larynx phonation area. ${ }^{2,3}$ The dorsolateral activation peak they report anterior/superior to the area for lip activation, appears to be the same dorsal throat area observed with stimulation. They postulated that the proximity of this intrinsic laryngeal musculature representation to the orofacial articulator representation might facilitate the tight coordination of multiple muscle groups needed for human speech. Rodel et al. were able to establish separable cortical representations of cricothyroid muscle (medially) and the vocalis muscle (laterally) by transcranial magnetic stimulation during electromyography. ${ }^{20}$ Our recent intracranial ECoG study demonstrating the cortical gamma activity associated with each speech articulator during a syllable articulation task also found peaks in cortical gamma activity in both dorsal and ventral larynx areas. ${ }^{1}$ Nearby, we observed a high probability of ECS-evoked vocalization, which appears to be centered in the same vicinity seen by Penfield. ${ }^{18}$ Activations in this region associated with both phonation and exhalation have been demonstrated with fMRI. ${ }^{13}$

Our probabilistic maps for lips, jaw, and tongue showed substantial overlap of the combined-population data, however the dorsal-ventral order of these motor and sensory groups in individuals was largely preserved. This ordered somatotopy with overlap is consistent with the findings in fMRI, ECoG, and microstimulation in primates. . $^{1,921}$ Similarly, the probability map for speech arrest in the pars opercularis and precentral gyrus fits well with prior results. 18,22

There are a number of limitations to the present study that should be mentioned. First, stimulation data are limited by craniotomy exposure. To account for this, our probabilities were calculated for $5 \times 5-\mathrm{mm}^{2}$ cortical bins, including only those patients stimulated in a given bin in the probability calculation. Second, observation, description, and subsequent classification of patient responses to stimulation based on visual information and patient reports allow for some possibility of misclassification. By iterative review of intraoperative video recordings we have attempted to minimize noise related to errors in patient response classification. Furthermore, the fact that our results correspond with those from fMRI studies in which subjects performed controlled tasks involving isolated muscles is reassuring. Finally, the spatial scale is coarse and there is no access to the sulci, where a significant portion of cortical representations also reside. Modern imaging studies do not have the power to establish causality in the same way that stimulation does, ${ }^{22}$ and therefore our findings can be an important reference for complementary noninvasive imaging studies. By monitoring for afterdischarges, we have ensured that the responses observed are not due to distant cortical spread of current.

\section{Conclusions}

In conclusion, we report probabilistic maps of function in the human vSMC based on direct electrical stimulation. We observed complex, stereotyped responses involving hand and orofacial musculature. The yield of specific responses could be highly variable across individuals; how- ever, the dorsal-ventral somatotopic order of representations was always maintained. The locations of maximal probability correspond well with prior studies of the somatotopy of the vSMC, including a bimodal representation of throat. These results define the ECS-derived functional map of the human vSMC.

\section{References}

1. Bouchard KE, Mesgarani N, Johnson K, Chang EF: Functional organization of human sensorimotor cortex for speech articulation. Nature 495:327-332, 2013

2. Brown S, Laird AR, Pfordresher PQ, Thelen SM, Turkeltaub P, Liotti M: The somatotopy of speech: phonation and articulation in the human motor cortex. Brain Cogn 70:31-41, 2009

3. Brown S, Ngan E, Liotti M: A larynx area in the human motor cortex. Cereb Cortex 18:837-845, 2008

4. Conant D, Bouchard KE, Chang EF: Speech map in the human ventral sensory-motor cortex. Curr Opin Neurobiol 24:63-67, 2014

5. Ferrier D: The localization of function in the brain. Proc $\mathbf{R}$ Soc Lond 22:228-232, 1873

6. Foerster O: The motor cortex in man in light of Hughlings Jackson's doctrine. Brain 59:135-159, 1936

7. Fritsch G, Hitzig E: Ueber die elektrische Erregbarkeit des Grosshirns. Arch Anat Physiol Wissen Med:300-332, 1870

8. Graziano MS, Taylor CS, Moore T: Complex movements evoked by microstimulation of precentral cortex. Neuron 34:841-851, 2002

9. Huang CS, Sirisko MA, Hiraba H, Murray GM, Sessle BJ: Organization of the primate face motor cortex as revealed by intracortical microstimulation and electrophysiological identification of afferent inputs and corticobulbar projections. J Neurophysiol 59:796-818, 1988

10. Jürgens U: Neural pathways underlying vocal control. Neurosci Biobehav Rev 26:235-258, 2002

11. Kuypers HG: An anatomical analysis of cortico-bulbar connexions to the pons and lower brain stem in the cat. J Anat 92:198-218, 1958

12. Kuypers HG: Some projections from the peri-central cortex to the pons and lower brain stem in monkey and chimpanzee. J Comp Neurol 110:221-255, 1958

13. Loucks TM, Poletto CJ, Simonyan K, Reynolds CL, Ludlow CL: Human brain activation during phonation and exhalation: common volitional control for two upper airway functions. Neuroimage 36:131-143, 2007

14. Meier JD, Aflalo TN, Kastner S, Graziano MS: Complex organization of human primary motor cortex: a high-resolution fMRI study. J Neurophysiol 100:1800-1812, 2008

15. Nii Y, Uematsu S, Lesser RP, Gordon B: Does the central sulcus divide motor and sensory functions? Cortical mapping of human hand areas as revealed by electrical stimulation through subdural grid electrodes. Neurology 46:360-367, 1996

16. Overduin SA, d'Avella A, Carmena JM, Bizzi E: Microstimulation activates a handful of muscle synergies. Neuron 76:1071-1077, 2012

17. Penfield W, Boldrey E: Somatic motor and sensory representation in the cerebral cortex of man as studied by electrical stimulation. Brain 60:389-443, 1937

18. Penfield W, Rasmussen T: Vocalization and arrest of speech. Arch Neurol Psychiatry 61:21-27, 1949

19. Penfield W, Welch K: Instability of response to stimulation of the sensorimotor cortex of man. J Physiol 109:358-365, 1949

20. Rödel RM, Olthoff A, Tergau F, Simonyan K, Kraemer D, Markus H, et al: Human cortical motor representation of the larynx as assessed by transcranial magnetic stimulation (TMS). Laryngoscope 114:918-922, 2004 
21. Takai O, Brown S, Liotti M: Representation of the speech effectors in the human motor cortex: somatotopy or overlap? Brain Lang 113:39-44, 2010

22. Tate MC, Herbet G, Moritz-Gasser S, Tate JE, Duffau H: Probabilistic map of critical functional regions of the human cerebral cortex: Broca's area revisited. Brain 137:27732782, 2014

23. Woolsey CN, Erickson TC, Gilson WE: Localization in somatic sensory and motor areas of human cerebral cortex as determined by direct recording of evoked potentials and electrical stimulation. J Neurosurg 51:476-506, 1979

\section{Author Contributions}

Conception and design: Chang, Breshears. Acquisition of data: Chang, Breshears. Analysis and interpretation of data: Chang, Breshears. Drafting the article: Chang, Breshears. Critically revis- ing the article: Breshears, Molinaro. Reviewed submitted version of manuscript: all authors. Statistical analysis: Breshears, Molinaro. Study supervision: Chang.

\section{Supplemental Information}

Previous Presentation

Portions of this work were presented in poster format at the

Congress of Neurological Surgeons Annual Meeting in San Francisco, CA, October 21, 2013

\section{Correspondence}

Edward F. Chang, Department of Neurological Surgery, University of California, San Francisco, 505 Parnassus Ave., M-779, San Francisco, CA 94143-0112. email: changed@neurosurg.ucsf.edu. 